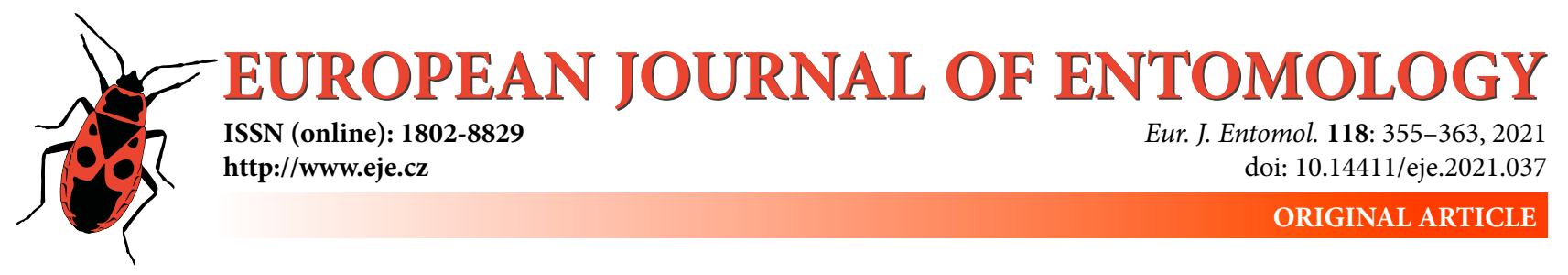

\title{
Characterization of the immune induced antimicrobial peptide in Drosophila melanogaster and Drosophila ananassae
}

\author{
Ramachandra Naik MEghashree iD and Kakanahalli NAGARAJ* iD \\ Department of Applied Zoology, Kuvempu University, Shankaraghatta, Shivamogga, 577451 Karnataka, India; \\ e-mails: meghanaik1992@gmail.com, knagarajv@gmail.com
}

Key words. Diptera, Drosophilidae, Drosophila, immune response, antimicrobial peptide, LC-MS/MS, cecropin A, haemolymph

\begin{abstract}
Insects can recognize invading pathogens and initiate an immune response. Among them, Drosophila has emerged as an invertebrate model for investigating innate immune responses in which antimicrobial peptides play a crucial role. In the present study, immune-induced antimicrobial peptides were characterized in $D$. melanogaster and $D$. ananassae using the agar well diffusion method, HPLC, SDS-PAGE and LC-MS/MS after infection with either S. aureus or E. coli. The HPLC revealed two and three differentially induced components, respectively, in $D$. melanogaster and $D$. ananassae flies infected with $S$. aureus and $E$. coli. The tricine SDS-PAGE analysis also revealed two and five differentially induced proteins, respectively, in $D$. melanogaster and $D$. ananassae infected with $E$. coli. In $E$. coli infected flies, the $\sim 6 \mathrm{kDa}$ band was produced at higher level. Based on LCMS/MS and Mascot analysis, the peptide was identified as a putative cecropin A-like peptide, and the data suggested that both species of Drosophila have exhibited a clear immune response. The flies were also able to discriminate between bacteria, as this putative cecropin A-like peptide was produced in flies infected with $E$. coli but not $S$. aureus.
\end{abstract}

\section{INTRODUCTION}

The fruit fly, Drosophila melanogaster has innate immunity against invading microbes. This includes both cellular and humoral immune responses (Lye, 2018; Meghashree \& Nagaraj, 2020). Antimicrobial peptides (AMPs) are an important component in the first line of defence (Yuchen et al., 2019). AMPs are endogenous peptides with a molecular weight (MW) of $\sim 2-22 \mathrm{kDa}$ and they are released by the fat body (analogue of the liver) into haemolymph to clear off the microbial infections (Troha et al., 2019). The interactions of AMPs with Gram-positive and Gramnegative bacteria differ. The positively charged AMPs selectively interact with prokaryotes having a negatively charged bacterial cell-wall, including lipopolysaccharides (LPS) and phospholipids. Based on the available data (FlyBase), nine distinct classes of AMPs (23 members) are identified in Drosophila (Thurmond et al., 2019). Among them, attacin, diptericin, cecropin and drosocin are produced in response to Gram-negative bacterial infections (Imd pathway), metchnikowin and defensin in response to a Gram-positive bacterial infection (Toll pathway) and drosomycin only in response to fungal infection (Sheehan et al., 2018).

As fruit flies are genetically similar in the way they combat diseases as humans, they can be used to evaluate mi- crobial infections and their associated immune responses (Baenas \& Wagner, 2019). Staphylococcus aureus (Grampositive) and Escherichia coli (Gram-negative) are two commonly used pathogens in studies on the immune response in Drosophila and are recognized by two distinct immune pathways. Like D. melanogaster, D. ananassae is another cosmopolitan and domestic species with high mutable property (Singh \& Yadav, 2015). Most of the studies on this species are on its behaviour, genetics and other evolutionary aspects (Kumar \& Singh, 2017; Kaladchibachi et al., 2019; Singh et al., 2020) in which there are no reports of infection-induced antimicrobial peptides. Understanding the immune response in this species and how it compares with that of $D$. melanogaster will provide a better knowledge of whether these two species differ in their microbe-induced immunity.

Cecropin A was first isolated from the moth, Hyalophora cecropia. Later on, cecropin-like peptides were identified in different insects belonging to Diptera, Hymenoptera, Coleoptera, Lepidoptera and Isoptera, and have different names, including sarcotoxin-I (Buonocore et al., 2021), hinnavin (Wu et al., 2018) and papiliocin (Kim et al., 2010), etc. In Drosophila, there are four cecropin genes on chromosome $3 \mathrm{R}$, which give rise to cecropin $\mathrm{A} 1$, cecropin A2, cecropin B and cecropin C peptides (Brian \& Clark,

\footnotetext{
* Corresponding author; e-mail: knagarajv@gmail.com
} 
2003), which are highly conserved in insects. There are also many research studies focused on identifying novel cecropin-like peptides in insects (Wu et al., 2015; Park \& Yoe, 2017; Manniello et al., 2021).

Hence, the objective of this study was to determine whether the induction of immune-induced AMP in D. melanogaster and D. ananassae flies infected with either $E$. coli or $S$. aureus differed. To authenticate its antimicrobial efficacy, homogenates of flies were tested for antibacterial activity in vitro. In addition, the phylogenetic relationships of the identified peptide are also discussed.

\section{MATERIALS AND METHODS}

\section{Fly stocks}

D. melanogaster (1.002) and D. ananassae (11.001) were reared on an instant Drosophila diet supplemented with yeast and kept at room temperature (RT) under $12 \mathrm{~L}: 12 \mathrm{D}$ conditions. The flies were obtained from the Drosophila Stock Center, University of Mysore, Mysore, Karnataka, India. For all experiments, 4-5 day old male and female adult flies $(1: 1)$ were used.

\section{Bacterial species}

Escherichia coli (MTCC 723) and Staphylococcus aureus (MTCC 7443) were obtained from MTCC, Chandigarh. All bacterial cultures were maintained on a nutrient agar medium. For liquid culture, bacteria were grown in sterile tubes containing 5 $\mathrm{mL}$ of nutrient broth (beef extract $-3 \mathrm{~g} / \mathrm{L}$; peptone $-0.5 \mathrm{~g} / \mathrm{L} ; \mathrm{NaCl}$ $-0.5 \mathrm{~g} / \mathrm{L}$ ), which was incubated for $24 \mathrm{~h}$ at $37^{\circ} \mathrm{C}$ before use. An optical density of $0.5\left(\mathrm{OD}_{600}\right)$ having $1 \times 10^{7} \mathrm{CFU} / \mathrm{mL}$ was used as an infectious dose and was obtained using a spectrophotometer (Multiskan Sky, Thermo scientific).

\section{Bacterial infection}

Flies were anesthetized and infected by inserting a tungsten needle into the lateral side of the thorax that had been dipped into either $S$. aureus or E. coli suspended in phosphate buffer saline (PBS). The treated flies were kept at RT by placing each of them in a fresh vial, laying the vial on its side until all flies recovered from the anesthesia in order to avoid the flies from becoming stuck in the food (Khalil et al., 2015). For the control group, flies were pricked with PBS dipped needle to create a non-septic injury.

\section{Preparation of crude extract and isolation of haemolymph}

Infected flies $(\mathrm{n}=50)$ were homogenized in $120 \mu \mathrm{L} 0.1 \%$ trifluoroacetic acid (TFA) at an ice-cold condition (Bhagavathula et al., 2017). The disrupted homogenate was further sonicated (QSonica 125, Thermo Scientific) at 20\% amplitude for 5 cycles at an interval of $5 \mathrm{~s}$. A $100 \mu \mathrm{L}$ supernatant of each extract was collected after centrifugation at $10000 \times \mathrm{g}$ for $15 \mathrm{~min}$ at $4^{\circ} \mathrm{C}$, freeze-dried using a lyophilizer (FreeZone, Labconco) for $10 \mathrm{~h}$ and stored at $-80^{\circ} \mathrm{C}$ until further use. These lyophilized samples were used for HPLC analysis and antibacterial activity.

The haemolymph was collected by means of centrifugation (Dhar \& Mishra, 2020) in which each fly after 24 h of bacterial infection was pricked with a needle to release the haemolymph. A $0.5 \mathrm{~mL}$ vial was punched with $4-5$ tiny holes using a $24 \mathrm{G}$ syringe needle and all the pricked flies $(n=50)$ were added to it. This vial was put inside a $1.5 \mathrm{~mL}$ microcentrifuge tube from which haemolymph was collected after centrifugation for $10 \mathrm{~min}$ at $2000 \times \mathrm{g}$ and stored at $-80^{\circ} \mathrm{C}$ (Damrau et al., 2015). From each vial, $2 \mu \mathrm{L}$ of haemolymph was extracted and freshly isolated samples were used for protein quantification and SDS-PAGE analysis.

\section{Protein quantification}

The protein concentration in the haemolymph from control and infected $(24 \mathrm{~h})$ flies was quantified in five independent experiments $(n=50)$ by direct concentration measurement in which the haemolymph was diluted $1: 5$ with double distilled water. A $2 \mu \mathrm{L}$ of diluted haemolymph was placed on a $\mu$ Drop $^{\mathrm{TM}}$ plate and absorbance was measured at $280 \mathrm{~nm}$ using Multiskan Sky spectrophotometer (Thermo Scientific ${ }^{\mathrm{TM}}$, USA). Protein concentration was determined based on this instrument's built-in protocol for the extinction coefficient of BSA.

\section{In vitro antibacterial activity determined using the agar well diffusion method}

The freeze-dried homogenate from Drosophila spp. obtained $24 \mathrm{~h}$ after injection with either $S$. aureus or E. coli or PBS (Control) were examined for antibacterial activity against the respective bacteria using the agar well diffusion method (Sewify et al., 2017). In brief, 5-mm diameter wells were made with a sterile cork borer (6 mm diameter) in nutrient agar plates spread with $S$. aureus or E. coli. The lyophilized homogenate $(5 \mathrm{mg})$ was suspended in $50 \mu \mathrm{L}$ PBS. Ciprofloxacin $(100 \mu \mathrm{g} / \mathrm{mL})$ was used as a standard. After incubation for $24 \mathrm{~h}$ at $37^{\circ} \mathrm{C}$, the zone of inhibition was measured in terms of its diameter in $\mathrm{mm}$.

\section{HPLC analysis}

The haemolymph from control and infected flies (24 h) was subjected to HPLC analysis. The lyophilized sample was dissolved in $0.1 \%$ TFA solution at a concentration of $25 \mathrm{mg} / \mathrm{mL}$. A $20 \mu \mathrm{L}$ of the crude sample was injected using a glass syringe into a $_{18}$ reverse-phase analytical column ( $5 \mu \mathrm{m}$ particle size; $250 \times$ $4.6 \mathrm{~mm}$ column) placed over an HPLC (Shimadzu). The solvent system included $0.1 \%$ TFA in Milli Q water (Solvent-A) and $80 \%$ aqueous Acetonitrile (ACN) with $0.1 \%$ TFA (Solvent-B) and the flow rate for the mobile phase was set at $1 \mathrm{~mL} / \mathrm{min}$. The elution was carried out with a linear gradient of $5-95 \%$ of solvent-B over a $60 \mathrm{~min}$ period. The eluted peaks were detected at $214 \mathrm{~nm}$ using an UV-DAD detector (SPD-M20A).

\section{SDS-PAGE analysis}

The profile of proteins in haemolymph was assessed by both Tris glycine (Laemmli, 1970) and tricine SDS-PAGE techniques (Schägger, 2006). A $30 \mu \mathrm{g}$ of haemolymph from control and infected flies $(24 \mathrm{~h})$ were placed in wells. For Tris-glycine, $4 \%$ stacking and $12 \%$ resolving gel were used and ran at $150 \mathrm{~V}$ for $75 \mathrm{~min}$. For tris-tricine SDS-PAGE, 4\% stacking, 10\% spacer and $16 \%$ resolving gel were used and ran at $150 \mathrm{~V}$ for $195 \mathrm{~min}$. To determine the MW, high (11-245 kDa; Himedia) and low range protein markers (3-45 kDa; SRL) were used. After electrophoresis, separated protein bands were detected by silver staining method (Gromova \& Celis, 2006). The density and number of bands were determined using Gelanalyzer software, version 19.1. The gel band of interest was cut out and placed in $1 \%$ acetic acid solution until required.

\section{Protein identification}

In-gel digestion

The piece of gel band was washed with $500 \mu \mathrm{L}$ of wash solution ( $50 \%$ acetonitrile, $50 \mathrm{mM}$ ammonium bicarbonate) and vortexed for $15 \mathrm{~min}$ at RT until it became opaque and stuck together. Then the gel band was spun down and the supernatant removed. A $3 \mathrm{~mL}$ of the Dithiothreitol solution was added to completely cover the piece of agar and incubated for $30 \mathrm{~min}$ at $56^{\circ} \mathrm{C}$ in an air thermostat. A $5 \mathrm{~mL}$ of Acetonitrile (ACN) was added, incubated for $10 \mathrm{~min}$ at RT and then the liquid was removed. A $3 \mathrm{~mL}$ of the iodoacetamide solution was added and incubated for $20 \mathrm{~min}$ at 
RT in the dark. The piece of agar was placed in acetonitrile and then centrifuged to remove all the liquid. Trypsin buffer (13 ng/ $\mu \mathrm{L}$ ) was added until the gel band was covered and then kept in an ice bucket for about $90 \mathrm{~min}$. A $1 \mu \mathrm{L}$ of ammonium bicarbonate buffer $(100 \mathrm{mM})$ was added to cover the gel band and keep them wet during enzymatic cleavage. $5 \%$ formic acid/ACN $(1: 2)$ was added and incubated for $15 \mathrm{~min}$. After centrifugation, the supernatant was transferred to a new vial. Then the samples were lyophilized and dissolved in $20 \mu \mathrm{L}$ of $2 \%$ acetonitrile $/ 0.1 \%$ formic acid solution.

\section{LC-MS/MS analysis}

The sample was analysed using an ultra-high-performance LC with mass selective detection and an Ultimate 3000 series LC (Dionex, USA) coupled with ESI tandem mass spectrometer (micrOTOF-Q II) (Bruker, Germany). A $3 \mu \mathrm{L}$ of sample was injected into LC precolumn (Pep map TM 100; $75 \mu \mathrm{m} \times 2 \mathrm{~cm}$; Nanoviper C18, $3 \mu \mathrm{m} ; 100 \AA$ ) and LC analytical column (EASY SPRAY PEPMAP RSLC C18 $3 \mu \mathrm{m} ; 50 \mathrm{~cm} \times 75 \mu \mathrm{m} ; 100 \AA)$ of an EASY-nLC 1200 LC instrument. Mobile phase A of $0.1 \%$ Formic acid in HPLC water and mobile phase B of $0.1 \%$ formic acid in acetonitrile was used. A linear gradient starting from 5\% to $95 \%$ in $60 \mathrm{~min}$ with an $0.2 \mathrm{~mL} / \mathrm{min}$ flow rate was recorded. The MS scan was carried within the $200-1800 \mathrm{~m} / \mathrm{z}$ range and the data acquired in MS/MS (auto) scanning mode.

\section{Mascot Search}

The data analysis was carried out using MASCOT search engine. In MS/MS ions search, the SwissProt database was used with all entries option for Taxonomy. The other parameters used included trypsin as a proteolytic enzyme, Cysteine carbamidomethylation as fixed modification, methionine oxidation as variable modification, the error window for peptide mass was $10 \mathrm{ppm}$ and fragment ion mass 0.6 Da. The decoy database was selected to calculate the false discovery rate. Only top rank peptide hits for given precursors were used for further protein identifications.

\section{Phylogenetic analysis}

The phylogenetic tree was constructed for seven cecropins: cecropin A (64 aa; P01507), A1 (63 aa; C0HKQ7), A2 (63 aa; C0HKQ8), B (63 aa; P14956) and C (63 aa; O16829), cecropin-2 (63 aa; XP_001955554.1) and sarcotoxin-1C (63 aa; XP_001955556.1) using the Maximum Likelihood method and JTT matrix-based model (Jones et al., 1992). The bootstrap consensus tree inferred from 1000 replicates was taken to represent the evolutionary history of the cecropins analysed. The percentage of replicate trees in which the associated cecropins clustered together in the bootstrap test was shown next to the branches (Felsenstein, 1985). Initial tree(s) for the heuristic search were obtained automatically by applying Neighbour-Joining and BioNJ algorithms to a matrix of pairwise distances estimated using the JTT model and then selecting the topology with the highest loglikelihood value. The evolutionary analyses were conducted in MEGA X v10.1.8 (Kumar et al., 2018).

\section{Statistical analysis}

The data were analysed using a two-way ANOVA for the protein concentration and antimicrobial activity with Bonferroni posthoc test using Graph pad Prism software 5.0. All the values are means \pm SEM. Values were considered significant when $* P<$ $0.05, * * P<0.01$ and $* * * P<0.001$.

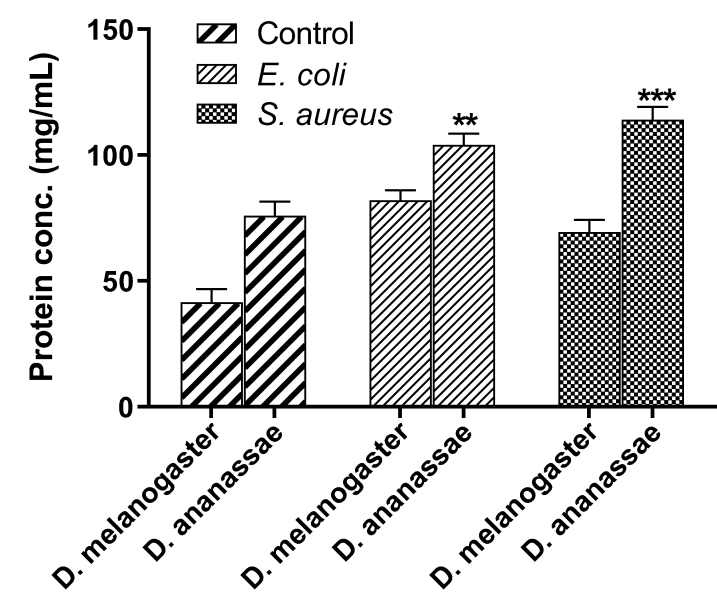

Fig. 1. Protein concentration in haemolymph. Mean protein concentration after infection of $D$. melanogaster $(n=50)$ and $D$. ananassae $(n=50)$ with either $S$. aureus or $E$. coli. Data presented as means \pm SEM. Significance compared to control. ${ }^{* *} P<0.05$ and ${ }^{\star * *} P<0.01$.

\section{RESULTS}

\section{The concentration of protein in haemolymph was greater in infected flies}

The average total concentration of protein in haemolymph based on five independent experiments was found to be significantly higher in both $D$. melanogaster and $D$. ananassae $24 \mathrm{~h}$ after infection with $E$. coli or $S$. aureus (Fig. 1). In D. melanogaster, infection with $E$. coli resulted in a significantly greater protein production (82.1 $\mathrm{mg} / \mathrm{mL} ; P<0.01)$ than infection with $S$. aureus $(69.4 \mathrm{mg} /$ $\mathrm{mL})$. However, in $D$. ananassae protein concentration was higher after infection with $S$. aureus $(114 \mathrm{mg} / \mathrm{mL})$ than $E$. coli $(104 \mathrm{mg} / \mathrm{mL})$. In addition, there is a very significant difference in total protein concentration in the two species Drosophila following bacterial infections.

\section{The Drosophila homogenate inhibits the growth of bacteria}

The freeze-dried homogenate of bacteria-infected flies inhibited the growth of bacteria differently as seen in the zone of inhibition against both $S$. aureus and E. coli compared with standard ciprofloxacin. An inhibition zone was not observed in PBS control flies. However, the degree of inhibition of bacteria is different. The $E$. coli infected $D$. melanogaster and $D$. ananassae homogenates resulted in a larger zone of inhibition than that of the $S$. aureus infected flies (Table 1 and Fig. S1).

\section{HPLC profile showed differential expression of immune induced molecules}

The HPLC profile of haemolymph showed that D. melanogaster and $D$. ananassae infected with either $S$. aureus or $E$. coli had two and three differentially produced molecules, respectively. This shows that there is a clear quantitative difference in the expression of AMPs. In addition, the elution time indicated (within $30 \mathrm{~min}$ ) that these molecules are possibly polar (Fig. 2). 
Table 1. The zones of inhibition for in vitro antibacterial activity. Zone of inhibition measured against $S$. aureus and $E$. coli for wholebody homogenate samples of $D$. melanogaster and $D$. ananassae injected, respectively, with the two bacteria. Ciprofloxacin antibiotic was used as a standard. Data are represented as means \pm SEM. Significance is shown compared to control. ${ }^{* *} P<0.001$.

\begin{tabular}{lccccc}
\hline \multirow{2}{*}{ Sample } & \multicolumn{3}{c}{ Zone of inhibition (diameter in mm) } \\
\cline { 2 - 3 } \cline { 5 - 6 } & \multicolumn{2}{c}{ D. melanogaster } & & \multicolumn{2}{c}{ D. ananassae } \\
\cline { 2 - 3 } \cline { 5 - 6 } PBS (Control) & $0.0 \pm 0.0$ & $0.0 \pm 0.0$ & & $0.0 \pm 0.0$ & $0.0 \pm 0.0$ \\
$\begin{array}{l}\text { Infected (E. coli } \\
\text { and S. aureus) }\end{array}$ & $11.1 \pm 0.15^{\star * *}$ & $10.0 \pm 0.10$ & & $11.5 \pm 4.50$ & $9.0 \pm 5.20$ \\
$\begin{array}{l}\text { Ciprofloxacin } \\
\text { (Standard) }\end{array}$ & $18.0 \pm 0.0$ & $21.0 \pm 2.00$ & & $18.0 \pm 0.10$ & $17.0 \pm 1.50$ \\
\hline
\end{tabular}

\section{Detection of a cecropin A-like peptide in E. coli infected flies using SDS-PAGE and LC-MS/MS analysis}

The SDS-PAGE analysis of haemolymph protein using the Tris-glycine method revealed several electrophoretic bands with MW ranging from $\sim 11-242 \mathrm{kDa}$, based on a densitometry analysis. Several proteins are up and downregulated during infection with either $E$. coli or $S$. aureus. Among them, three $(58,34$, and $13 \mathrm{kDa})$ and five proteins $(45,33,27,14$, and $11 \mathrm{kDa})$ were markedly produced in D. melanogaster and D. ananassae, respectively (Fig. 3).

As the Tris-glycine method doesn't resolve the low MW peptides, haemolymph samples from $D$. melanogaster and $D$. ananassae infected with $S$. aureus or $E$. coli were separated using the tris-tricine method. The data showed that a single protein band of $\sim 6 \mathrm{kDa}$ (based on the retention factor calculated using gelanalyzer software) was differentially produced in both $D$. melanogaster and $D$. ananassae injected with $E$. coli, but not with $S$. aureus or PBS (Fig. 4A). In addition, one protein band in D. melanogaster and four protein bands within $14 \mathrm{kDa}$, were highly produced in D. ananassae infected with E. coli (Fig. 4B) and these protein bands were not recorded in the two species of flies
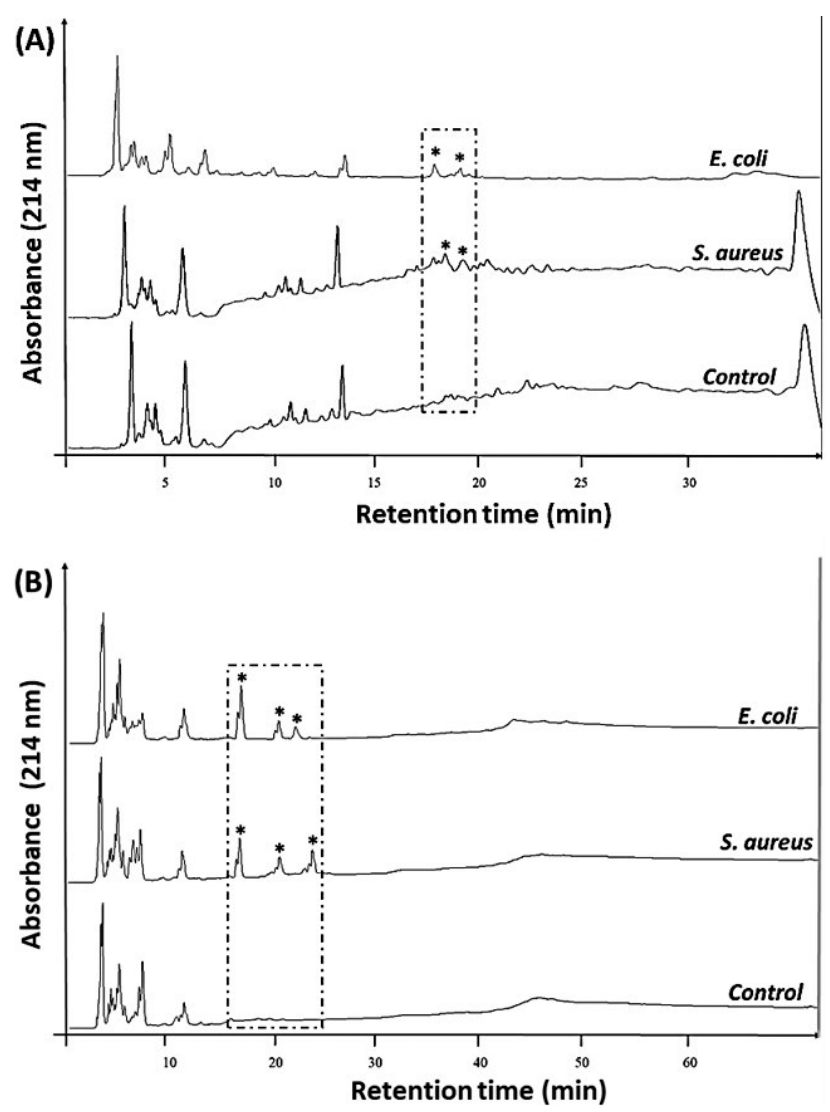

Fig. 2. HPLC chromatogram of Drosophila haemolymph after infection. The HPLC profile of haemolymph obtained from (A) $D$. melanogaster and (B) D. ananassae, $24 \mathrm{~h}$ post-injection with PBS (control), E. coli or $S$. aureus show two and three differently expressed molecules, respectively. ${ }^{*}$ Peaks of immune-induced components.

infected with $S$. aureus. The data also showed that the control groups of these two flies have distinct protein profiles. The higher molecular weight protein bands were also differentially produced in the E. coli infected flies. Here,
(A)

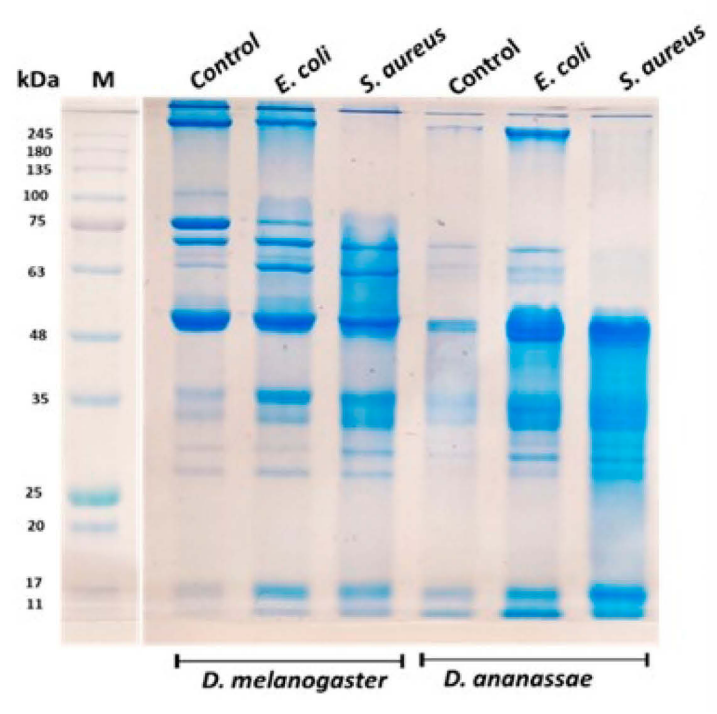

(B)

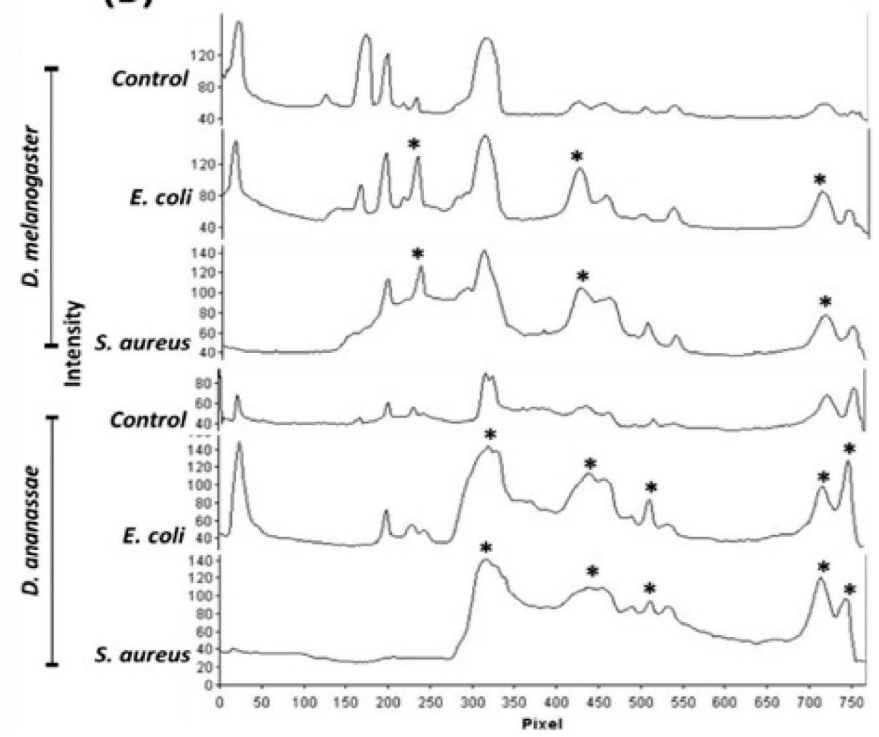

Fig. 3. Tris-glycine SDS PAGE and densitometry analysis. (A) The Tris-glycine SDS-PAGE analysis of the haemolymph and (B) its densitometry analysis. ${ }^{*}$ Differentially induced proteins. $\mathrm{M}$ - Marker. 


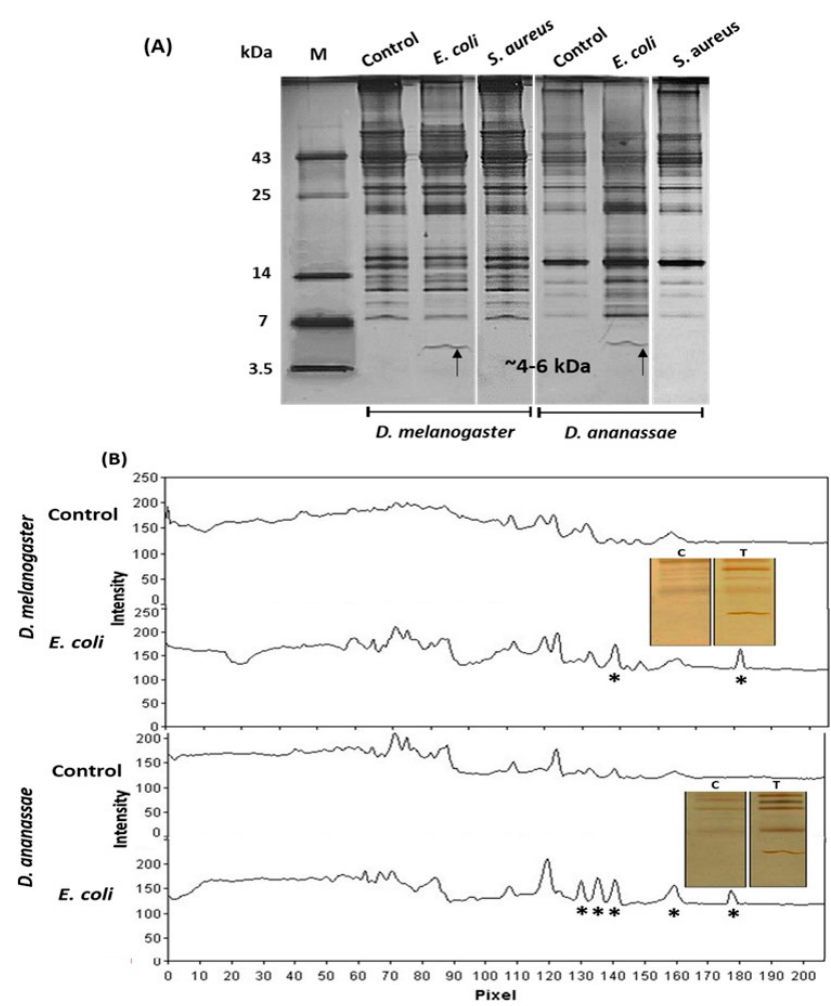

Fig. 4. Tris-tricine SDS PAGE and densitometry analysis. (A) The Tris-tricine SDS-PAGE analysis of the haemolymph from Drosophila infected with $S$. aureus or $E$. coli showed one differentially expressed peptide of $\sim 6 \mathrm{kDa}$ against $E$. coli infection. (B) The densitometry analysis showed two and five immune induced proteins in $E$. coli infected $D$. melanogaster and $D$. ananassae flies, respectively. ${ }^{*}$ Differentially induced proteins. M - Marker; C - Control haemolymph and $\mathrm{T}-$ treated haemolymph of $E$. coli.

D. melanogaster and D. ananassae infected with E. coli showed three and two highly produced proteins, respectively, compared to PBS injected flies (Fig. 5).

The Mascot search of the LC-MS/MS analysis of a protein band $(\sim 6 \mathrm{kDa})$ from both $E$. coli infected $D$. melanogaster and $D$. ananassae gave a fragment ion with sequence AGPAVAVVGQATQIAK, which is similar to cecropin A of 6952 Da with a protein sequence coverage of $26 \%$ (Table 2 and Fig. 6). The mass spectrometry proteomics data are deposited in the 'ProteomeXchange Consortium' via the PRIDE (Perez-Riverol et al., 2019) partner repository with the dataset identifier PXD025417 with DOI: 10.6019/ PXD025417 for D. melanogaster and PXD025419 with DOI: 10.6019/PXD025419 for D. ananassae.

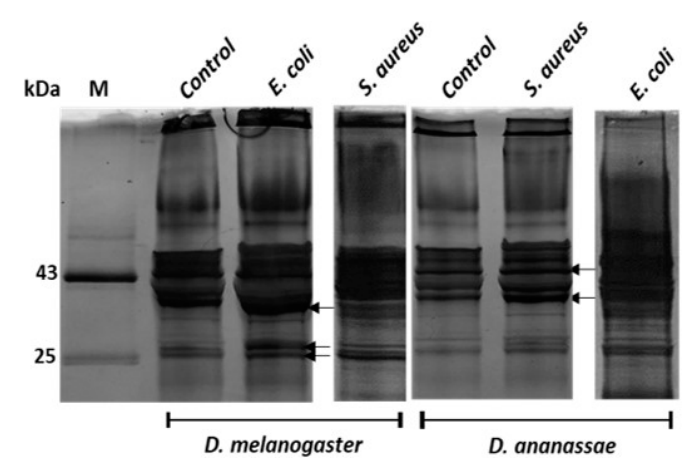

Fig. 5. Tris-tricine SDS PAGE for high-range proteins. The Tristricine SDS-PAGE analysis for high-range proteins of the haemolymph from $D$. melanogaster and $D$. ananassae infected with $E$. coli showed three and one differentially expressed proteins, respectively (Arrow); M - Marker.

\section{Phylogenetic analysis of the putative cecropin A-like peptide sequence}

Cecropins occurred in insects before the divergence of Diptera, Lepidoptera and Coleoptera. The phylogenetic tree (Fig. 7A) indicate that Drosophila cecropins are present in one branch and Hyalophora's cecropin in another branch. The result of a multiple sequence alignment and phylogenetic analysis indicate that the putative cecropin A-like peptide has an approximately $11.7 \%$ sequence similarity with other Drosophila cecropins (Fig. 7B). The result also support the independent evolution of the cecropin peptide family in these insects (Tassanakajon et al., 2015).

\section{DISCUSSION}

Studies on the immune system of insects help in understanding the complexity of the immune system and the vital role of AMPs in insects' innate immunity. Drosophila has been a suitable model for studying the role of antimicrobial peptides in neutralizing circulating pathogens. The measurement of protein concentration in complex mixtures other than cell lysates can be better assessed at $280 \mathrm{~nm}$. The increased total protein level recorded could be due to bacterial infection resulting in a higher metabolic rate in these flies. The type of signal generated by $E$. coli could be stronger and have resulted in a higher metabolic rate and hence increased total protein level in $E$. coli infected flies than in $S$. aureus infected flies.

The agar well diffusion method is commonly used for screening the antibacterial activity of AMPs. Here, a putative cecropin A-like peptide produced due to bacterial infection could be the reason for the antimicrobial activity recorded against bacterial pathogens. In a recent study (Park $\&$ Yoe, 2017), the minimum inhibitory and bactericidal

Table 2. Mascot search results. Protein identification obtained using Mascot software search of corresponding gel bands from $D$. melanogaster and $D$. ananassae.

\begin{tabular}{|c|c|c|c|c|c|c|c|}
\hline $\begin{array}{l}\text { Source } \\
\text { of protein }\end{array}$ & $\begin{array}{c}\text { LC-MS/MS } \\
\text { detected sequence }\end{array}$ & $\begin{array}{l}\text { Observed } \\
\text { mass (Da) }\end{array}$ & $\begin{array}{l}\text { Mascot } \\
\text { score }\end{array}$ & $\begin{array}{l}\text { Protein } \\
\text { identified }\end{array}$ & $\begin{array}{c}\text { Uniprot } \\
\text { acession no. }\end{array}$ & $\begin{array}{l}\text { Mass } \\
(\mathrm{Da})\end{array}$ & $\begin{array}{c}\text { Sequence } \\
\text { ceverage }(\%)\end{array}$ \\
\hline $\begin{array}{l}\text { Drosophila } \\
\text { melanogaster } \\
\text { Drosophila } \\
\text { ananassae }\end{array}$ & $\begin{array}{c}\text { AGPAVAV } \\
\text { VGQATQI } \\
\text { AK }\end{array}$ & 740.9282 & 111 & $\begin{array}{l}\text { Cecropin-A } \\
\text { (similar to } \\
\text { Hyalophora } \\
\text { cecropia) }\end{array}$ & P01507 & 6952 & 26 \\
\hline
\end{tabular}




\section{(A) MNFSRIFFFVFACLTALAMVNAAPEPKWKLFKKIEKVGQNIRDGIIKAGPAVAVVGQATQIAKG}
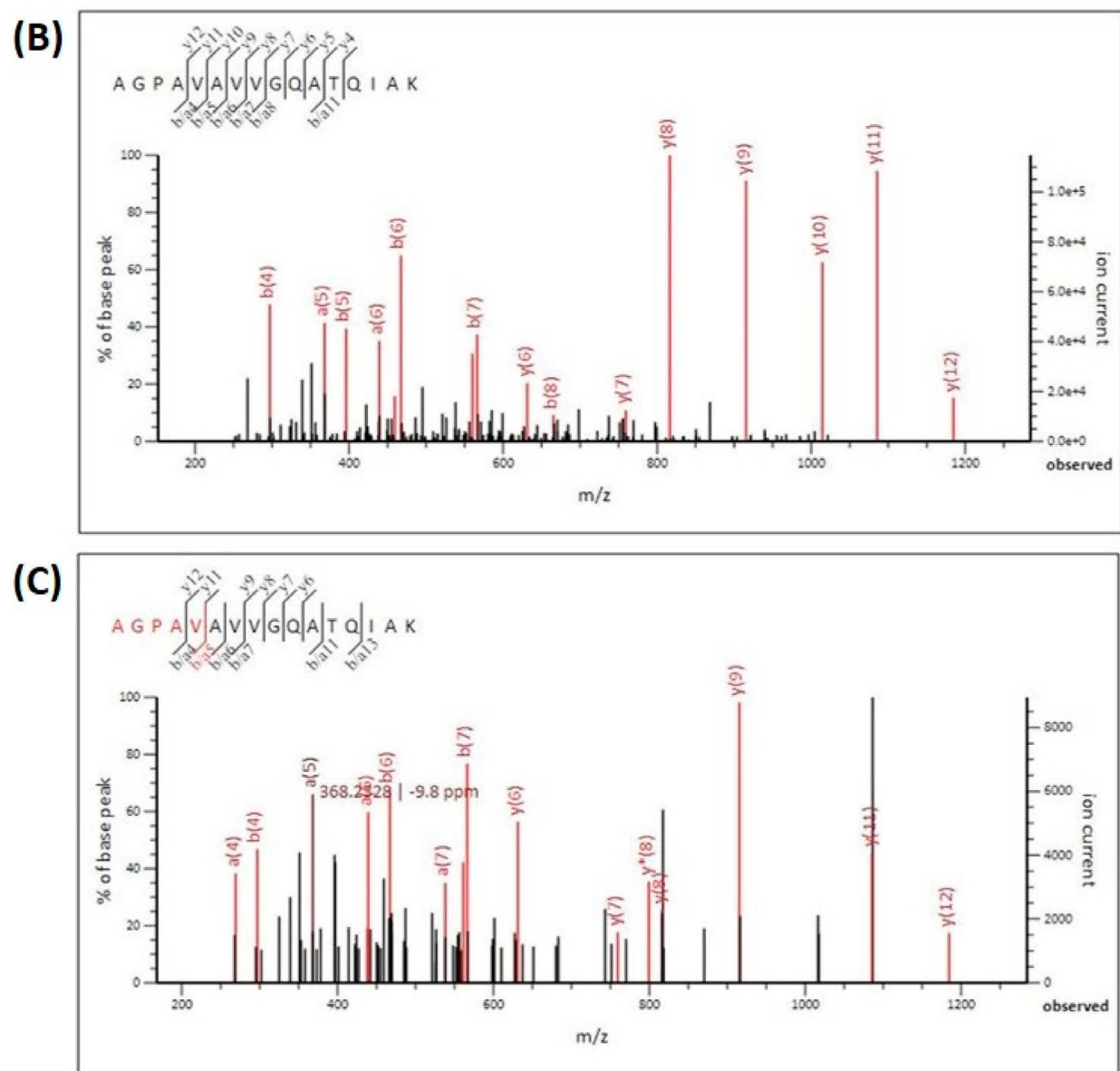

Fig. 6. Amino acid sequence and fragmentation spectra of the tryptic peptide. (A) The full sequence of cecropin-A in which the red coloured sequence is the LC-MS/MS identified tryptic peptide and its spectra sourced from the haemolymph of (B) D. melanogaster and (C) D. ananassae.

concentrations evaluated for a cecropin-like peptide has higher antibacterial effects against Gram-negative bacteria. In the $S$. aureus infection, though a cecropin A-like peptide was not recorded, there were other immune-induced proteins with higher MWs, which could also account for the in vitro antimicrobial activity recorded in these infections. In addition, the HPLC profile of haemolymph also confirms the presence of immune-induced components in flies infected with $S$. aureus.

Drosophila is considered to be good a model for understanding the variability in conserved genes expressed in closely related species (Hodgins-Davis et al., 2009). The tricine SDS-PAGE method has been less used for identifying immune-induced AMPs in Drosophila, but as shown here, can clearly be used to identify low MW peptides. The production of a specific AMP against $E$. coli by both flies indicates that inducible immune genes may have been conserved in these two flies (Hanson et al., 2016). AMPs are not produced in uninfected or PBS-pricked flies (Li et al., 2019; Feng et al., 2021; Kapila et al., 2021). They are not constitutively expressed in Drosophila haemolymph. Further, the level of induced immunity was stronger against $E$. coli than $S$. aureus. This is in accordance with earlier reports in which several AMPs are elicited against Gram- negative bacterial infections, but not non-flagellated Grampositive bacterial infections (Lemaitre et al., 1997). In another report, cecropin is strongly expressed in Drosophila cell lines by bacterial lipopolysaccharide and flagellin, but weakly by peptidoglycan (Samakovlis et al., 1992). This confirms that $E$. coli can elicit a stronger immune response than S. aureus in Drosophila spp. Thus, as in the mammalian immune system, where different pattern recognition receptors are involved in the identification of lipopolysaccharide (TLR-4) and peptidoglycan (TLR-2), Drosophila might be able to discriminate the different compositions of the membranes of Gram-positive and Gram-negative bacteria (Takeuchi et al., 1999).

Among several antimicrobial peptides of Drosophila origin, cecropin A, a $4.3 \mathrm{kDa}$ peptide (active form), which was first isolated from the haemolymph of the Lepidopteran H. cecropia (Mylonakis et al., 2016) is mainly expressed during Gram-negative bacterial infections (Wen et al., 2019), as it is an $\alpha$-helical antimicrobial peptide that mainly kills Gram-negative bacteria (Fu et al., 2004). Diptericin $(9 \mathrm{kDa})$ and drosocin $(2.19 \mathrm{kDa})$ are also produced in response to Gram-negative bacterial infections. In this study, the putative cecropin A-like peptide expression was 


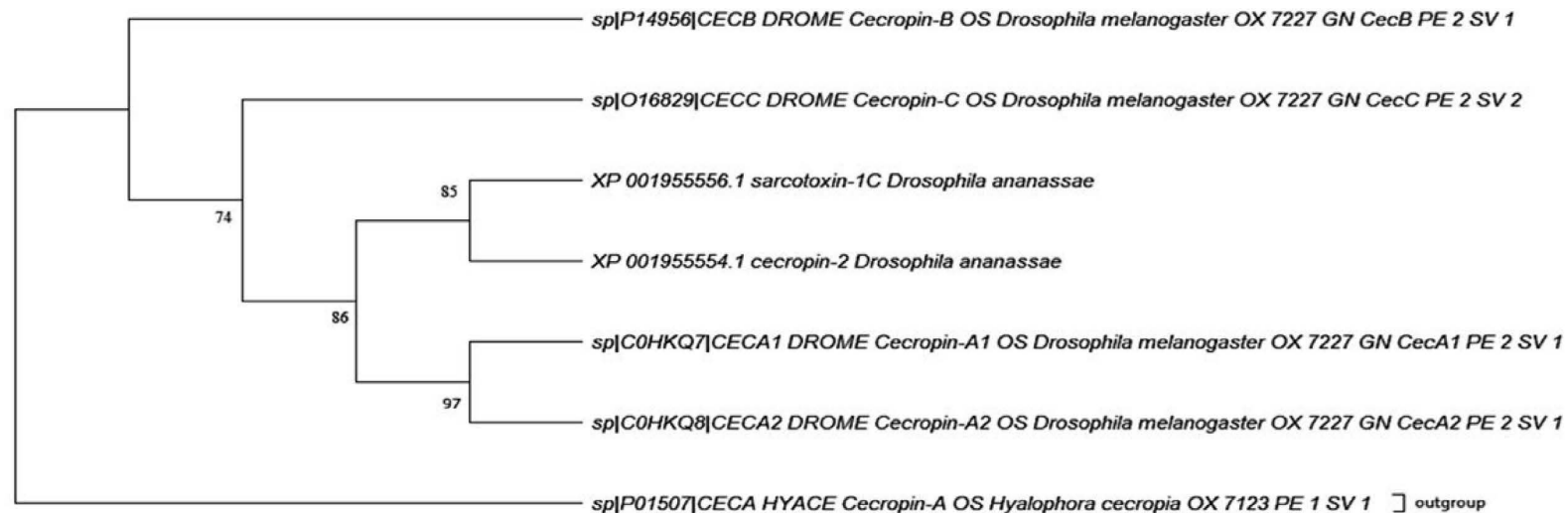

(B)

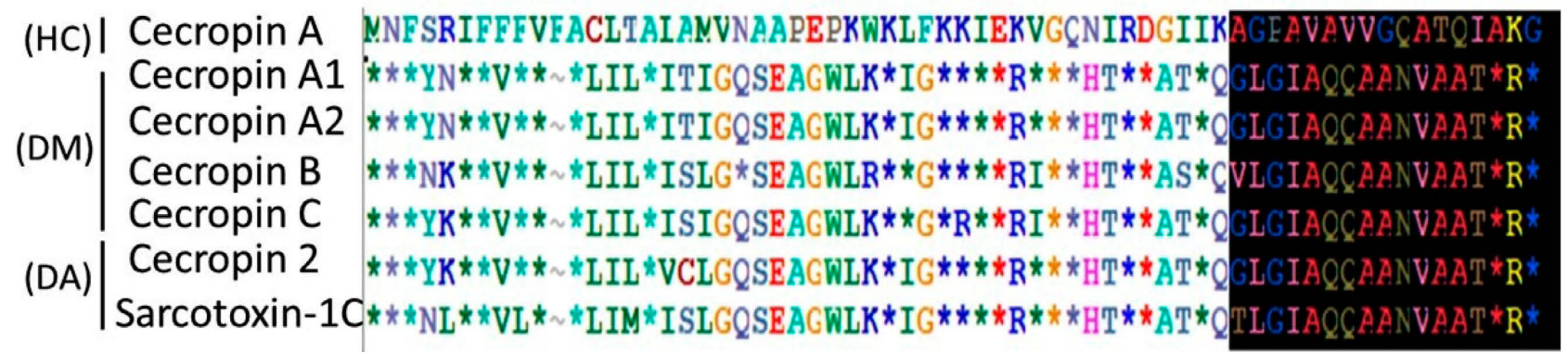

Fig. 7. (A) Phylogenetic analysis of seven cecropins. The phylogram of the amino acid sequence of cercopins from Drosophila melanogaster (4), D. ananassae (2) and $H$. cecropia (1) was generated in MEGA X v10.1 using Maximum Likelihood method with 1000 replications of the bootstrapping analysis. Outgroup and the scientific names are labelled with Uniprot accession no. (B) Multiple sequence alignment of cecropins. Sequence alignment of full amino acid sequence of cecropin from $H$. cecropia (HA) with that of $D$. melanogaster (DM) and D. ananassae (DA). The amino acid sequences (AGPAVAVVGQATQIAKG) identified from LC-MS/MS and Mascot analysis on the black background indicate other cecropins. Identical residues are indicated by *.

recorded only in haemolymph from Drosophila infected with E. coli.

We have shown marked similarities and some notable differences in the immune responses to bacterial infection by $D$. melanogaster and $D$. ananassae. Though the protein profiles were different in these two species, the AMP was recorded in both species in response to infection with $E$. coli, but not $S$. aureus. Previous research also indicates that many of the differentially expressed genes in D. melanogaster during the parasitoid-specific immune response have similar transcriptional responses in other closely related species of Drosophila. The gene expression profiles in D. melanogaster and D. simulans are very similar (Salazar-Jaramillo et al., 2017). In addition, 83\% of proteincoding genes in D. ananassae are homologous to those in D. melanogaster (Uniprot, 2021a), which could explain the similar protein patterns recorded in SDS gels of the two species studied.

In this study, the LC-MS/MS-based mascot analysis detected a peptide with 16 amino acid sequences (AGPAVAVVGQATQIAK), which is similar to the cecropin-A from $H$. cecropia, with a good Mascot score and sequence coverage. However, the identified putative cecropin A-like peptide has only $37-41 \%$ similarity with other Drosophila cecropins (Uniprot, 2021b). Hence, the present finding of a cecropin A-like peptide is an addition to the pool of already known cecropins, produced by Lepidoptera like $H$. cecropia, Bombyx mori, Dipteran Musca domestica and Coleop- teran Acalolepta luxuriosa, etc. In addition, as a part of the evolutionary link, the Lepidopteran cecropin is similar to those reported for Diptera. For example, the cecropin from Brachycera (Diptera) is closely related to the Lepidopteran cecropin (Brady et al., 2019) and the mosquito cecropin (Diptera) is more similar to the B. mori cecropinD (Lowenberger et al., 1999). Notwithstanding, that the remaining unidentified region of this putative cecropin A-like peptide may have a similar or different amino acid sequence to the Hyalophora's cecropin. There are many other cecropin like peptides, such as sarcotoxin-I (Buonocore et al., 2021), papiliocin (Kim et al., 2010), stomoxyn (Boulanger et al., 2002b; Landon et al., 2006) and hinnavin (Yoe et al., 2006), etc.

Further genomic analysis could validate this peptide as either cecropin A or its ortholog in Drosophila. The detection of a particular cecropin in several species of Diptera, like Drosophila, is not uncommon. Cecropin B is produced by both $D$. sechellia and $D$. simulans, and cecropin C by $D$. takahashii, D. simulans and D. sechellia (Uniprot, 2021b). Similarly, in this study, a putative cecropin A-like peptide was detected in both $D$. melanogaster and D. ananassae. This data support the tree topology as the obtained sequence is most similar to $H$. cecropia. In other words, each sequence is more similar to its ortholog in another species than to other members in the same species. That cecropinA1, A2, B, C are conserved in D. melanogaster indicates that both paralogs are from duplication of a cecropin-like 
peptide ancestor. The homology of the sequences of cecropin $\mathrm{A}(H$. cecropia $)$ and cecropins $\left(\mathrm{A}_{1}, \mathrm{~A}_{2}, \mathrm{~B}\right.$, and $\left.\mathrm{C}\right)$ from D. melanogaster, indicate that the detected peptide region of cecropin A has two amino acids similar to known cecropins of $D$. melanogaster and $D$. ananassae.

This study has shown how different bacterial infections generate distinct immune responses in different genetic backgrounds. This study is novel as there are no reports on the evaluation of immune responses in $D$. ananassae and this is the only report of the production of putative cecropin A-like peptide in Drosophila spp.

\section{CONCLUSION}

This study has shown there is an antibacterial immune response in $D$. melanogaster and $D$. ananassae against $S$. aureus and E. coli. The production of a putative cecropin A-like peptide in both species of Drosophila is reported here for the first time against infection with $E$. coli, but not against $S$. aureus. The role of this peptide in innate immunity with comparison to other paralogues needs to be investigated.

ACKNOWLEDGEMENTS. The authors are thankful to DST, New Delhi, India for providing financial support through the INSPIRE FELLOWSHIP program (Order No. DST/INSPIRE/2016/ IF160813 dated 5/10/2016). We also thank N.B. Thippeswamy, Dept. of Microbiology, Kuvempu University, for allowing us to use his laboratory facilities for carrying out some of the experiments. The authors also acknowledge the Proteomics facility, Indian Institute of Science, Bangalore for conducting the LC-MS/ MS analysis.

AUTHOR'S CONTRIBUTION. KN developed the concept, idea and supervised the experimental design. MRN performed the experiments and wrote the manuscript. $\mathrm{KN}$ edited the manuscript and approved it.

CONFLICT OF INTEREST. The authors do not have any conflict of interest.

\section{REFERENCES}

Baenas N. \& Wagner A.E. 2019: Drosophila melanogaster as an alternative model organism in nutrigenomics. - Genes Nutr. 14: $1-11$

Bhagavathula N., Meedidoddi V., Bourque S., Vimaladevi R., Kesavakurup S., Selvadurai D., Shrivastava S. \& Krishnappa C. 2017: Characterization of two novel antimicrobial peptides from the cuticular extracts of the ant Trichomyrmex criniceps (Mayr), (Hymenoptera: Formicidae). - Arch. Insect Biochem. Physiol. 94(4): e21381, 8 pp.

Boulanger N., Munks R.J., Hamilton J.V., Vovelle F., Brun R., Lehane M.J. \& Bulet P. 2002: Epithelial innate immunity. A novel antimicrobial peptide with antiparasitic activity in the blood-sucking insect Stomoxys calcitrans. - J. Biol. Chem. 277: 49921-49926.

Brady D., Grapputo A., Romoli O. \& Sandrelli F. 2019: Insect cecropins, antimicrobial peptides with potential therapeutic applications. - Int. J. Mol. Sci. 20(23): 5862, 22 pp.

LAzZARo B.P. \& Clark A.G. 2003: Molecular population genetics of inducible antibacterial peptide genes in Drosophila melanogaster. - Mol. Biol. Evol. 20: 914-923.
Buonocore F., Fausto A.M., Della Pell G., Roncevic T., Gerdol M. \& Picchiettr S. 2021: Attacins: A promising class of insect antimicrobial peptides. - Antibiotics 10(2): 212, 12 pp.

Damrau C., Toshima N., Tanimura T., Brembs B. \& Colomb J. 2018: Octopamine and tyramine contribute separately to the counter-regulatory response to sugar deficit in Drosophila. Front. Syst. Neurosci. 11: 100, 11 pp.

Dhar G. \& Mishra M. 2020: Comet assay to detect the severity of DNA damage in Drosophila. In Mishra M. (ed.): Fundamental Approaches to Screen Abnormalities in Drosophila. Springer Nature, pp. 87-96.

Felsenstein J. 1985: Confidence limits on phylogenies: An approach using the bootstrap. - Evolution 39: 783-791.

Feng M., Xia J., Fei S., Peng R., Wang X., Zhou Y., Wang P., SwEVERS L. \& SUN J. 2021: Identification of silkworm hemocyte subsets and analysis of their response to baculovirus infection based on single-cell RNA sequencing. - Front. Immunol. 12: $645359,16 \mathrm{pp}$.

Fu H., Bürrstad A., Dahlgren C. \& Bylund J. 2004: A bactericidal cecropin-A peptide with a stabilized alpha-helical structure possess an increased killing capacity but no proinflammatory activity. - Inflammation 28: 337-343.

Gromova I. \& Celis J.E. 2006: Protein detection in gels by silver staining: a procedure compatible with mass-spectrometry, in cell biology. In Celis J.E., Carter N., Hunter T., Shotton D., Simons K. \& Small J.V. (eds): Cell Biology. A Laboratory Handbook. Vol. 4. Academic Press, San Diego, CA, pp. 219-223.

Hanson M.A., Hamilton P.T. \& Perlman S.J. 2016: Immune genes and divergent antimicrobial peptides in flies of the subgenus Drosophila. - BMC Evol. Biol. 16: 228, 14 pp.

Hodgins-Davis A. \& Townsend J.P. 2009: Evolving gene expression: from G to E to GxE. — Trends Ecol. Evol. 24: 649-658.

Jones D.T., TAYLoR W.R. \& ThorNTON J.M. 1992: The rapid generation of mutation data matrices from protein sequences. CABIOS 8: 275-282.

Kaladchibachi S., Secor M.A., Negelspach D.C. \& Fernandez F. 2019: Longitudinal study of sleep and diurnal rhythms in Drosophila ananassae. — Exp. Gerontol. 116: 74-79.

Kapila R., Kashyap M., Poddar S., Gangwal S. \& Prasad N.G.G. 2021: Evolution of pathogen-specific improved survivorship post-infection in populations of Drosophila melanogaster adapted to larval crowding. — PLOS ONE 16(4): e0250055, $11 \mathrm{pp}$.

Khalil S., Jacobson E., Chambers M.C. \& Lazzaro B.P. 2015: Systemic bacterial infection and immune defense phenotypes in Drosophila melanogaster. - J. Vis. Exp. 99: e52613, 9 pp.

Kim S.R., Hong M.Y., Park S.W., Choi K.H., Yun E.Y., Goo T.W., Kang S.W., Suh H.J., KIm I. \& Hwang J.S. 2010: Characterization and cDNA cloning of a cecropin-like antimicrobial peptide, papiliocin, from the swallowtail butterfly, Papilio $x u$ thus. - Mol. Cells 29: 419-423.

Kumar S. \& Singh A.K. 2017: Population genetics of Drosophila: Genetic variation and differentiation among Indian natural populations of Drosophila ananassae. - Zool Stud. 56: 1, 10 pp.

Kumar S., Stecher G., Li M., Knyaz C. \& Tamura K. 2018: MEGA X: Molecular evolutionary genetics analysis across computing platforms. - Mol. Biol. Evol. 35: 1547-1549.

LAEMMLI U.K. 1970: Cleavage of structural proteins during the assembly of the head of bacteriophage T4. - Nature 227: 680-685.

Landon C., Meudal H., Boulanger N., Bulet P. \& Vovelle F. 2006: Solution structures of stomoxyn and spinigerin, two 
insect antimicrobial peptides with an alpha-helical conformation. - Biopolymers 81: 92-103.

Lemaitre B., Reichhart J.M. \& Hoffmann J.A. 1997: Drosophila host defense: differential induction of antimicrobial peptide genes after infection by various classes of microorganisms. - Proc. Natl. Acad. Sci. U.S.A. 94: 14614-14619.

Li T., YAN D., Wang X., Zhang L. \& Chen P. 2019: Hemocyte changes during immune melanization in Bombyx mori infected with Escherichia coli. — Insects 10(9): 301, 15 pp.

Lowenberger C., Charlet M., Vizioli J., Kamal S., Richman A., Christensen B.M. \& Bulet P. 1999: Antimicrobial activity spectrum, cDNA cloning, and mRNA expression of a newly isolated member of the cecropin family from the mosquito vector Aedes aegypti. — J. Biol. Chem. 274: 20092-20097.

Lye S.H. \& Chtarbanova S. 2018: Drosophila as a model to study brain innate immunity in health and disease. - Int. $J$. Mol. Sci. 19(12): 3922, 14 pp.

Manniello M.D., Moretta A., Salvia R., Scieuzo C., Lucchetti D., Vogel H., Sgambato A. \& Falabella P. 2021: Insect antimicrobial peptides: potential weapons to counteract the antibiotic resistance. - Cell. Mol. Life Sci. 78: 4259-4282.

Meghashree R.N. \& Nagaraj K. 2020: Evaluation of antibacterial immune response in Drosophila melanogaster and Drosophila ananassae. — Indian J. Exp. Biol. 11: 751-759.

Mylonakis E., Podsiadlowski L., Muhammed M. \& Vilcinskas A. 2016: Diversity, evolution and medical applications of insect antimicrobial peptides.-Phil. Trans. R. Soc. Lond. (B) 371: 20150290, $11 \mathrm{pp.}$

PARK S. \& Yoe S.M. 2017: A novel cecropin-like peptide from black soldier fly, Hermetia illucens: Isolation, structural and functional characterization. - Entomol. Res. 47: 115-124.

Perez-Riverol Y., Csordas A., Bai J., Bernal-Llinares M., Hewapathirana S., Kundu D.J., Inuganti A., Griss J., MaYer G., EISENAChER M., ET AL. 2019: The PRIDE database and related tools and resources in 2019: improving support for quantification data. - Nucl. Acids Res. 47: D442-D450.

Salazar-Jaramillo L., Jalvingh K.M., HaAn A., KraAiJeveld K., Buermans H. \& Wertheim B. 2017: Inter- and intra-species variation in genome-wide gene expression of Drosophila in response to parasitoid wasp attack. - BMC Genomics 18: 331, $14 \mathrm{pp}$.

Samakovlis C., Asling B., Boman H.G., Gateff E. \& Hultmark D. 1992: In vitro induction of cecropin genes - an immune response in a Drosophila blood cell line. - Biochem. Biophys. Res. Commun. 188: 1169-1175.

SCHÄGGER H. 2006: Tricine-SDS-PAGE. - Nat. Protoc. 1: 16-22. Sewify G.H., Hamada H.M. \& AlhadRami H.A. 2017: In vitro evaluation of antimicrobial activity of alimentary canal ex-

\section{D. melanogaster}
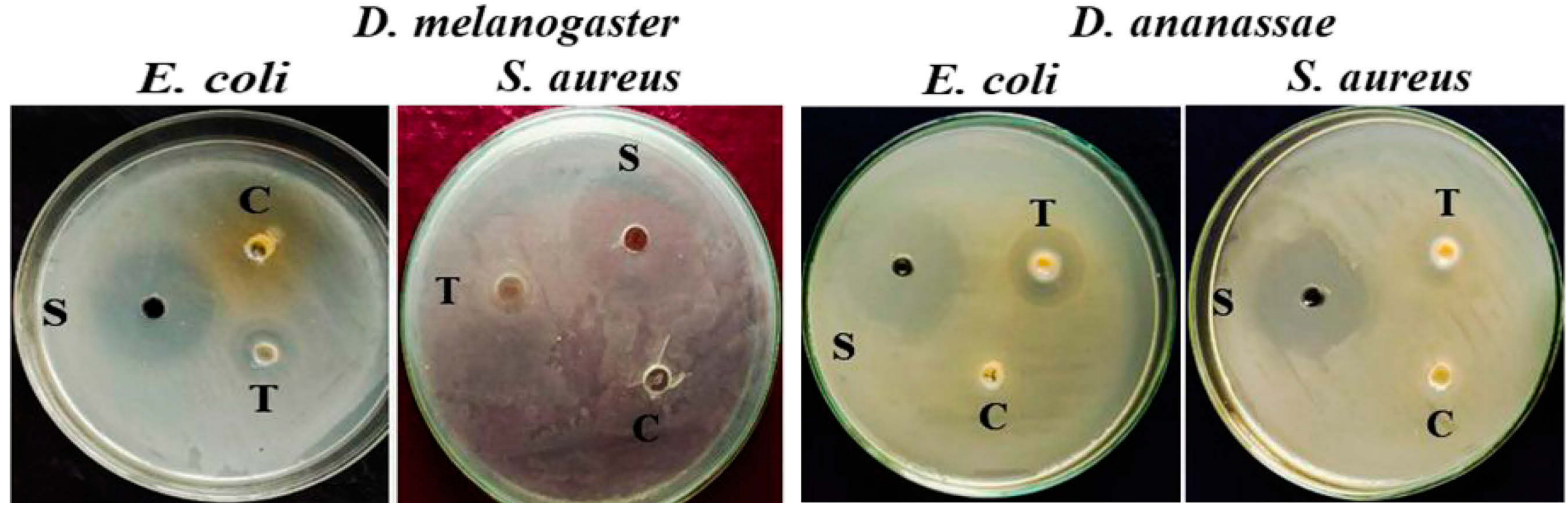

Fig. S1. Antibacterial activity of the Drosophila homogenate. The whole-body homogenates from both species of fly infected with either S. aureus or E. coli had shown the zone of inhibition. C - Control (PBS); T - Treated; S - Standard (ciprofloxacin). 